\section{Commentary: Expertise in thoracoabdominal aortic aneurysm repair-More than just the knife}

\author{
Ali Hage, MD, ${ }^{a}$ John Bozinovski, MD, MSc, ${ }^{b}$ \\ Maral Ouzounian, $\mathrm{MD}, \mathrm{PhD},{ }^{\mathrm{c}}$ and \\ Michael W. A. Chu, MD, MEd, an behalf of the \\ Canadian Thoracic Aortic Collaborative (CTAC)
}

Thoracoabdominal aortic aneurysm (TAAA) repair remains a formidable operation in which few surgical teams develop mastery. Coselli and colleagues ${ }^{1}$ have the world's largest experience with TAAA repair and have been pioneers in this field, having set the bar for all other programs with decades of experience and excellent patient outcomes. Despite major advancements in surgical technique and their unparalleled experience, however, TAAA repair remains associated with substantial rates of adverse events, ranging from $10 \%$ to $19 \%$. $^{1}$ In their 2-part article in this issue of the Journal, Chatterjee and colleagues ${ }^{2,3}$ illustrate that provision of excellent outcomes for patients undergoing TAAA repair is much more than just the operation itself, rather encompassing the entire perioperative journey of the patient.

Most surgical innovation tends to originate from a few leading centers of expertise where, fortunately, contemporary knowledge translation and early adoption methods have enabled widespread dissemination of these surgical techniques worldwide. It has been easy to focus on the technical aspects of TAAA repair, sometimes overshadowing the critically important perioperative care measures and comprehensive, multidisciplinary teams required to achieve

From ${ }^{\mathrm{a}}$ Western University, London, Ontario, Canada; ${ }^{\mathrm{b}}$ The Ohio State University Wexner Medical Center, Columbus, Ohio; and the ${ }^{c}$ University of Toronto, Toronto, Ontario, Canada.

Disclosures: M.W.C. has received speaker's honoraria from Medtronic, Edwards Lifesciences, Terumo Aortic, Boston Scientific, and Abbott Vascular. All other authors have nothing to disclose with regard to commercial support.

Received for publication Dec 8, 2019; accepted for publication Dec 8, 2019; available ahead of print Feb 26, 2020.

Address for reprints: Michael W. A. Chu, MD, MEd, B6-106 University Hospital, London Health Sciences Centre, 339 Windermere Rd, London, Ontario N6A 5A5, Canada (E-mail: Michael.Chu@lhsc.on.ca).

J Thorac Cardiovasc Surg 2021;161:706-7

$0022-5223 / \$ 36.00$

Copyright (c) 2020 by The American Association for Thoracic Surgery

https://doi.org/10.1016/j.jtcvs.2019.12.130

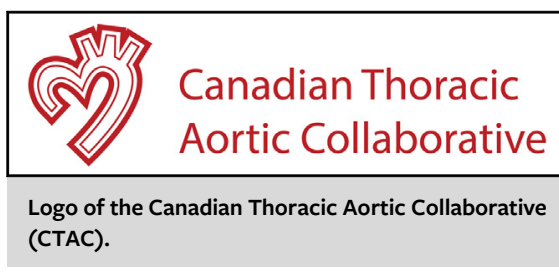

\author{
CENTRAL MESSAGE \\ An excellent thoracoabdominal \\ aortic aneurysm repair is only \\ half the story; equally important \\ are key preoperative and post- \\ operative care measures that ul- \\ timately define optimal patient \\ outcomes.
}

excellent patient outcomes. This knowledge and care gap is likely further exacerbated in complex, multisystem operations such as TAAA repair, for which surgical volumepatient outcomes relationships are well described. ${ }^{4}$ The Enhanced Recovery After Surgery (ERAS) initiative promotes and disseminates standardized protocols and wellfounded recommendations to improve the perioperative management of cardiac surgery patients. ${ }^{5}$ These strategies can dramatically improve patient outcomes, and this effect will be likely magnified in TAAA repair because of the magnitude of the operation, the increased perioperative risk, and the susceptibility of end organs to hemodynamic insults during and after surgery.

TAAA repair results in major physiologic derangements, even in the best hands. Every organ system is affected by the surgery, and at least temporary perioperative dysfunction in multiple organ systems is common. The 4 key factors that limit the translation of these insults into long-term morbidities and mortality are as follows: (1) limiting the intraoperative duration of the insult, (2) expertise and knowledge in preventing complications, (3) how quickly these adverse events are correctly diagnosed and treated, and (4) how well the organs are optimized before the surgery. First and foremost, excellent surgical skills and techniques are the central determining factors of good postoperative outcomes. In addition to diligent surgery, there is an absolute need for a large comprehensive, multidisciplinary team to provide optimal preoperative and postoperative care to prevent and manage the complications with the use of standardized tools and protocols. Most importantly, this multidisciplinary team should also be responsible for screening and optimizing the patient's status before the 
surgery, thereby preventing the occurrence of complications and improving both postoperative outcomes and cost savings. ${ }^{5}$

Developing a sophisticated multidisciplinary team for the preoperative and postoperative care of patients undergoing TAAA repair requires a significant infrastructure and support. Substantial contribution is required from cardiology, anesthesia, perfusion, nursing, intensive care, and allied health disciplines, such as physiotherapy, speech language pathology, and pharmacy. Despite their disparate roles, each team member has an important influence on the patient's final outcome.

In summary, as exquisitely described by Chatterjee and colleagues $^{2,3}$ for the Coselli's team in their recent publications, becoming an expert in TAAA repair certainly requires excellent surgical skills in the operating room. Perhaps equally importantly, it also necessitates the presence of an outstanding multidisciplinary team that is efficient and excellent in optimizing the patients before surgery and also vigilant in recognizing and treating their complications afterward.

\section{References}

1. Coselli JS, LeMaire SA, Preventza O, de la Cruz KI, Cooley DA, Price MD, et al Outcomes of 3309 thoracoabdominal aortic aneurysm repairs. J Thorac Cardiovasc Surg. 2016;151:1323-37.

2. Chatterjee S, Casar JG, LeMaire SA, Preventza O, Coselli JS. Perioperative care after thoracoabdominal aortic aneurysm repair: the Baylor College of Medicine experience. Part 1: preoperative considerations. J Thorac Cardiovasc Surg. 2021;161:693-8.

3. Chatterjee S, Casar JG, LeMaire SA, Preventza O, Coselli JS. Perioperative care after thoracoabdominal aortic aneurysm repair: the Baylor College of Medicine experience. Part 2: postoperative management. J Thorac Cardiovasc Surg. 2021; 161:699-705.

4. Cowan JA Jr, Dimick JB, Henke PK, Huber TS, Stanley JC, Upchurch GR Jr. Surgical treatment of intact thoracoabdominal aortic aneurysms in the United States: hospital and surgeon volume-related outcomes. J Vasc Surg. 2003;37: 1169-74.

5. Engelman DT, Ben Ali W, Williams JB, Perrault LP, Reddy VS, Arora RC, et al. Guidelines for perioperative care in cardiac surgery: enhanced recovery after surgery society recommendations. JAMA Surg. May 4, 2019 [Epub ahead of print]. 